\title{
Automatic Detection and Severity Assessment of Pepper Bacterial Spot Disease via MultiModels Based on Convolutional Neural Networks
}

\author{
Qiufeng Wu, Northeast Agricultural University, China \\ (iD) https://orcid.org/0000-0002-4787-2549 \\ Miaomiao Ji, Northeast Agricultural University, China \\ Zhao Deng, Northeast Agricultual University, China
}

\begin{abstract}
Pepper bacterial spot disease caused by Xanthomonas campestris is the most common pepper bacterial disease, which ultimately reduces productivity and quality of products. This work uses deep convolutional neural networks (CNNs) to serve fine-grained pepper bacterial spot disease severity classification tasks. The pepper bacterial spot disease leaf images collected from the PlantVillage dataset are further annotated by botanists and split into healthy samples (label1), general samples (label2), and serious samples (label3). To extract more effective and discriminative features, an integrated neural network denoted as MultiModel_VGR is proposed for automatic detection and severity assessment of pepper bacterial spot disease, which is based on three powerful and popular deep learning architectures, namely VGGNet, GoogLeNet and ResNet. Compared with state-ofthe-art single CNN architectures and binary-integrated MultiModels, MultiModel_VGR yields the best overall accuracy of $95.34 \%$ on the hold-out test dataset, which may have great potential in crop disease control for modern agriculture.
\end{abstract}

\section{KEYWORDS}

Convolutional Neural Networks, Deep Learning, Disease Severity Assessment, Pepper Disease Detection, Xanthomonas Campestris

\section{INTRODUCTION}

Pepper, as one of the most widely cultivated economical crop throughout the world, plays an important role in the world's agricultural production and trade. For decades, pepper has been severely affected by various diseases, such as red anthracnose, bacterial spot and black mold, etc. Among these diseases, pepper bacterial spot disease caused by Xanthomonas campestris is the most common pepper bacterial disease, which can ultimately reduce productivity and quantitative losses in crop yield. A very small number of diseased leaves tend to spread the infection to the whole batch of crops 
and cause epidemic all over the field, which is undoubtedly devastating. Timely information on the harm degree of crop disease can guide agricultural producers to take necessary and more targeted measures to avoid additional financial losses. Therefore, it is urgent to develop an early and accurate method for pepper bacterial spot disease detection and severity assessment.

The existing method for crop diseases detection and severity assessment is simply naked eye observation by farmers in the field with the guidance of plant pathologists, whose process of diagnosis is not only subjective but also time-consuming and laborious. Advances in science and technology now make it possible for computer vision approaches to assist us in automatic detection and severity assessment of crop diseases tasks. CNNs can locate important features itself and automatically learn appropriate features from training dataset instead of manual feature extraction. They have such excellent generalization ability and robustness that they excel in diverse areas, such as signal processing (Xie et al., 2017), road crack detection (Zhang et al., 2016) and biomedical image analysis (Zhou et al., 2017). CNNs also have been successfully applied in the field of agriculture, including diagnosis of crop diseases (Zhang et al., 2019a, 2019b), recognition of weeds (Philipp et al., 2018), selection of fine seeds (Wang \& Cheng, 2016), pest identification (Cheng et al., 2017), fruit counting (Rahnemoonfar \& Sheppard, 2017) and land cover classification (Kussul et al., 2017), etc. Although remarkable performances have been achieved in normal crop diseases classification, it is still hard to distinguish diseases with subtle discrimination. Compared with classification among different crop diseases, pepper bacterial spot disease severity extent classification is much more challenging, as there exist large intra-class similarity and small inter-class variance in pathological symptoms. As shown in Figure 1, in the early stage of infection, Xanthomonas campestris bacterium attack the pepper leaf causing one or several light-colored spots. The serious indication of the disease on leaflets consists of a large number of brownish lesions which are not delimited in size and enlarge rapidly under favourable conditions.

Fine-grained image classification refers as discriminating the sub-categories sharing one common basic-level category through digital images (Sumbul et al., 2017). Pepper bacterial spot disease detection and severity assessment belongs to the category of fine-grained classification task and our schedule is aimed to directly boost up the accuracy through more effective and discriminative features and representations extracted by an effective algorithm. Inspired by the great success of CNNs-based methods in image classification as well as other applications, the integrated model in our work, denoted as MultiModel_VGR, is just based on the three powerful and popular deep learning architectures,

Figure 1. Illustration of the pepper bacterial spot disease: healthy samples, general samples and serious samples
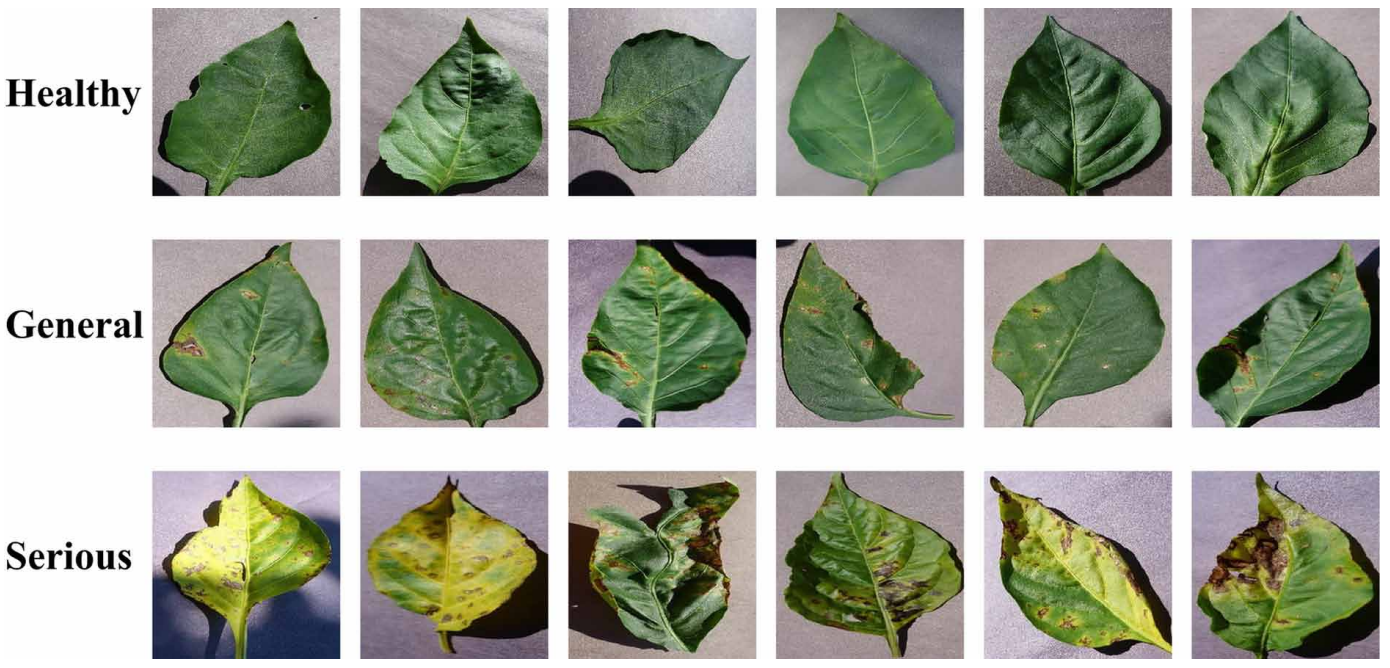
namely VGGNet (Simonyan \& Zisserman, 2014), GoogLeNet (Szegedy et al., 2014) and ResNet (He et al., 2015), where the input data is propagated forward to each basic single model and then input to the classifier after concatenation. In addition, it is easier to fuse the features extracted from the convolutional layers of each single model by way of transfer learning.

The purpose of this paper is to realize detection and severity assessment of pepper leaf disease infected by Xanthomonas campestris using MultiModel_VGR based on CNNs with higher accuracy, lower error rate and less training duration. The rest of this paper is organized as follows. Section 2 starts with an overview of related works. Section 3 proposes the MultiModel_VGR of VGGNet, GoogLeNet and ResNet. Section 4 is dedicated to the conducted experiments and wraps up the paper with a discussion of our major findings. Finally, Section 5 concludes this paper and limitations and future work have been mentioned.

\section{RELATED WORKS}

Traditional approaches for plant diseases detection and severity assessment were used to following pipelined procedures of image segmentation, feature extraction and pattern recognition. Li et al. (2012) proposed a method based on K-means clustering segmentation of the pepper disease images and a SVM classifier was designed based on thirty-one effective selected features to identify pepper diseases. Test recognition rates of identifying pepper downy mildew and pepper powdery mildew reached 90\% and $93.33 \%$, respectively. Han et al. (2015) developed a novel computer vision-based approach for automatically identifying crop diseases based on marker-controlled watershed segmentation, superpixel based feature analysis and classification. Their experimental result demonstrated that their proposed approach can accurately detect crop diseases and assess the disease severity with efficient processing speed. Although progress has been achieved on recognition of plant diseases, hand-engineered components require considerable time and manpower and sometimes manual search for good feature extractors is not an easy and obvious task. Selecting and extracting the optimal features of visible disease symptoms makes an expensive effort that depends on experts' knowledge and could not be generalized well.

Not until the advent of deep learning was this less of a problem. CNNs are famous for end-toend training mode and have demonstrated success in crop diseases identification. Zhang et al. (2019) used deep learning method to identify peach leaf disease infected by Xanthomonas campestris. In comparison to SVM, KNN and BPNN, AlexNet (Krizhevsky et al., 2012) got the best accuracy of $100 \%$. Mohanty et al. (2016) trained a deep CNN based on pre-trained AlexNet and GoogLeNet from a public dataset of 26 classes of diseased and healthy plant leaves. GoogLeNet achieved an accuracy of $99.35 \%$ on a hold-out test dataset. Geetharamani and Arun Pandian (2019) proposed a nine-layer deep CNN model to effectively classify 38 distinct classes of healthy and diseased plants using leaf images and further discussed the influence of data augmentation, training epochs, batch size and dropout. Guan et al. (2017) trained a series of shallow networks trained from scratch and deep models fine-tuned by transfer learning to diagnose the severity of fine-grained apple black rot disease. VGGNet16 model trained with transfer learning yielded the best overall accuracy of $90.4 \%$ on the hold-out test dataset.

All the literature aforementioned used single CNN architecture to identify the crop diseases. Although the basic framework of CNNs, such as AlexNet, VGGNet, GoogLeNet and ResNet, have been mature enough and widely used in various fields, single model can't meet the fine-grained image classification requests. More robust discriminative feature extraction and representation for subordinate categories discrimination is one of effective methods for dealing with fine-grained image classification tasks. Liang et al. (2019) developed an automatic image-based PD2SE-Net (Plant Disease Diagnosis and Severity Estimation Network) for multi-function computer-aided diagnosis system with capabilities of plant species recognition, disease classification, and disease severity estimation. Residual structure and shuffle units were combined to achieve stronger classification performance. 
Lin et al. (2019) proposed a unified CNN model, denoted as M-bCNN, based on convolutional kernel matrix, for fine-grained wheat leaf diseases classification. Gains of data streams, neurons, and link channels made their approach demonstrate promising performances when competing against plain networks including AlexNet and VGGNet. Extracting discriminative features through incorporating structural units in state-of-the-art CNNs is a simple and instinctive idea. Yu et al. (2018) presented FFCMNET (Feature Fusion based Car Model Classification Net) for fine-grained car model classification based on CNN and feature fusion. Separate branches including UpNet and DownNet were designed for extracting the features of upper parts and lower parts of the car frontal images separately. Their scheme shown favorable results on a benchmark dataset and outperformed the state-of-the-art models in the classification of large datasets. The well-known Inception-ResNet-v2 (Szegedy et al., 2016) was also born out of the fusion of two excellent deep CNNs, as the name suggests, and has been widely applied in various classification tasks.

Inspired by network in network principle and integrated concept, feature fusion from pre-trained $\mathrm{CNN}$ frameworks is used to overcome the limitations of a single CNN model and make recognition decision. Improved performance can be observed of the integrated method over single ones.

\section{MATERIALS AND METHODS}

\subsection{Dataset Description and Image Preprocessing}

The pepper bacterial spot disease dataset comes from an open access repository of images on plant health, namely PlantVillage (Hughes et al., 2015), including healthy samples and diseased samples infected by Xanthomonas Campestris. Each single diseased pepper leaf is annotated by botanists according to the disease harm degree and diseased samples are further divided into general and serious. To the end, healthy samples (label1), general samples (label2) and serious samples (label3) hold the number of 494, 458 and 420 respectively as shown in Table 1 . Train-validation-test scheme is applied in our work. Healthy, general and serious images with the number of 100 respectively are selected as test dataset which are used for predicting and evaluating the performance of the models and the rest images are used for training. The training dataset is further split into training dataset (80\%) and validation dataset (20\%) to determine whether the model is over-fitting. To the end, the training dataset is composed of 857 samples in total, validation dataset is 215 samples in total and test dataset is 300 samples in total.

Since the samples in the PlantVillage dataset are arbitrarily sized RGB images, data preprocessing is necessary and all the images are resized to the expected input size of the respective networks, i.e. $224 \times 224$ for VGGNet and ResNet, and 229×229 for GoogLeNet. Model optimization and prediction are performed on these rescaled images. Given the large amount parameters of the utilized deep CNNs, artificial expanding our utilized dataset is necessary as it can both minimize the possibility of over-fitting and better the robustness of network. Data augmentation techniques which include rotation, flipping, shearing, zooming, shifting and colour changes are randomly performed in training phase as shown in Figure 2.

Table 1. Details of pepper bacterial spot disease dataset

\begin{tabular}{|l|c|c|c|c|}
\hline \multicolumn{1}{|c|}{ Label } & Training Samples & Validation Samples & Test Samples & Total \\
\hline Healthy (label1) & 315 & 79 & 100 & 494 \\
\hline General (lable2) & 286 & 72 & 100 & 458 \\
\hline Serious (lable3) & 256 & 64 & 100 & 420 \\
\hline Total & 857 & 215 & 300 & 1372 \\
\hline
\end{tabular}


Figure 2. Data augmentation procedure of pepper leaf images
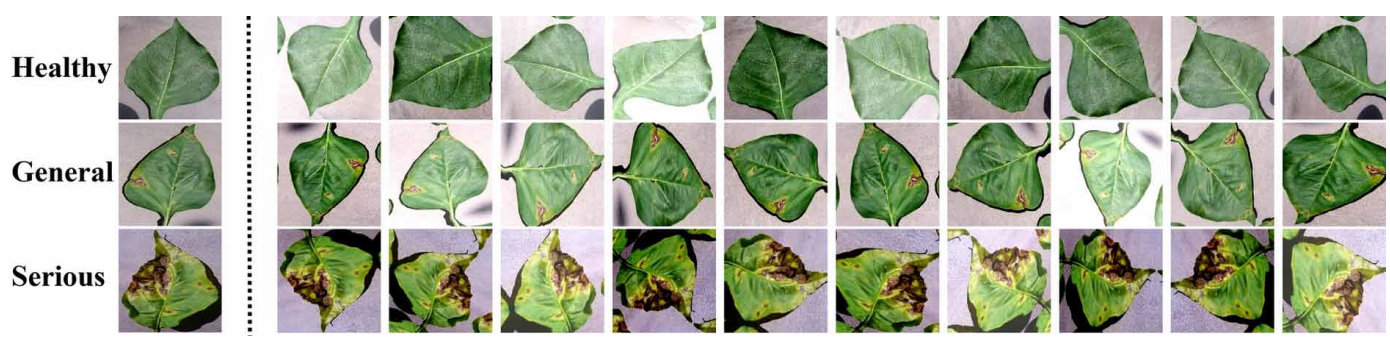

\subsection{Identification Model of Pepper Leaf Disease Infected by Xanthomonas Campestris}

This paper uses CNNs to identify the pepper leaf disease infected by Xanthomonas campestris, which can be abstracted as a multi-class classification problem. The classification process of this work can be described as shown in Figure 3. Assuming a mapping function from pepper leaf images to corresponding predicted labels (i.e. healthy (label1), general (label1) and serious (label2)): $f: X \rightarrow Y$. Then the pepper bacterial spot disease training dataset is denoted as $\left\{\left(x_{i}, y_{i}\right) \mid i=1,2, \cdots n, y_{i} \in\{0,1,2\}\right\}$. The training dataset is input into the algorithm $A$ (i.e. CNN architecture) and the parameters in CNN are updated continuously to minimize the average loss function, which is defined as:

$$
J(\omega)=-\frac{1}{n} \sum_{i=1}^{n} y_{i} \log \left(h_{\omega}\left(x_{i}\right)\right)
$$

Figure 3. The mathematical model of pepper leaf disease detection and severity assessment

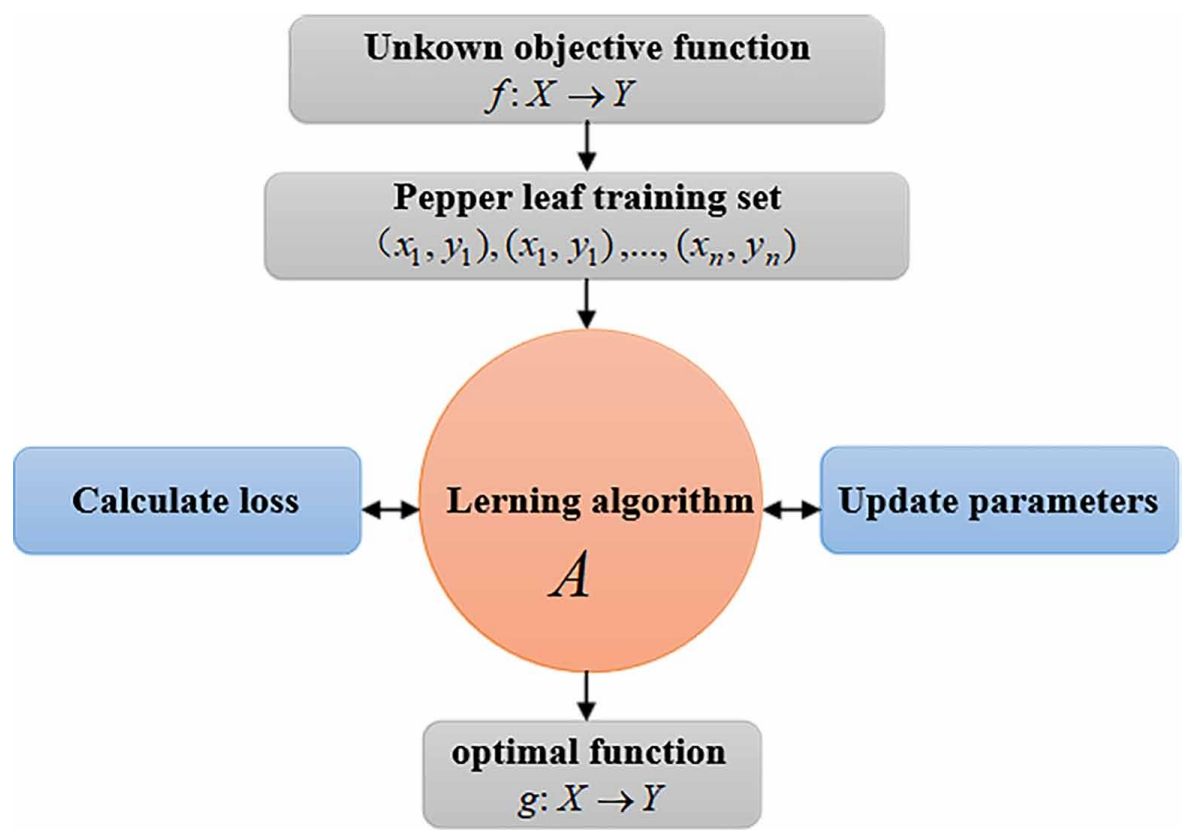


where $x_{i}$ represents the $i t h$ input data and $n$ is the number of input data referred to as batch size, $y_{i}$ represents the true label of the $i$ th input data, and $h_{\omega}\left(x_{i}\right)$ is the predicted label of the ith input data depending on the current weights $\omega$. During learning the CNN model, the end-to-end optimization can be performed using the stochastic gradient descent (SGD) method for the loss function.

Noted that $\omega$ in convolutional layers are set to the pre-trained network weights and not changed and SGD algorithm is used to learn the best set of weights and biases of the added layers that minimize the loss function. After a series of epochs, a relatively optimal function is obtained, which is the optimal function: $g: X \rightarrow Y$. The test dataset can be input into the optimal function and predicted labels will be obtained.

\subsection{MultiModeI_VGR: Fusion of VGGNet, GoogLeNet and ResNet}

Deep learning is currently a very active research field in computer vision and image classification. A typical deep CNN consists of an input and an output or classification layer, as well as multiple hidden layers. The hidden layers of a CNN typically consist of convolutional layers, pooling layers, fully connected layers and sometimes softmax layer. Most CNNs architectures follow the design pattern of Lenet-5 architecture (Lecun et al., 1998). Thereafter, a number of architectures have been designed. In this work, an integrated model called MultiModel_VGR is proposed for the task of automatic detection and severity assessment of pepper bacterial spot disease, which include the state-of-the-art CNNs: VGGNet, GoogLeNet and ResNet.

\subsubsection{VGGNet}

VGGNet (Simonyan \& Zisserman, 2014) improved performance considerably by increasing the CNN depth. Although VGGNet seemed a little bit violent, it did work, which earned the first runner-up in ILSVRC 2014. VGGNet is at the leading level of extensibility and generalization in transfer learning tasks. VGGNet16 as the basic network of the MultiModel_ VGR involves 13 convolutional layers with very small receptive fields $(3 \times 3)$, five max-pooling layers of size $2 \times 2$, three fully-connected layers, and a linear layer with softmax activation in the output (see Figure 4).

\section{Figure 4. The architecture of VGGNet16}

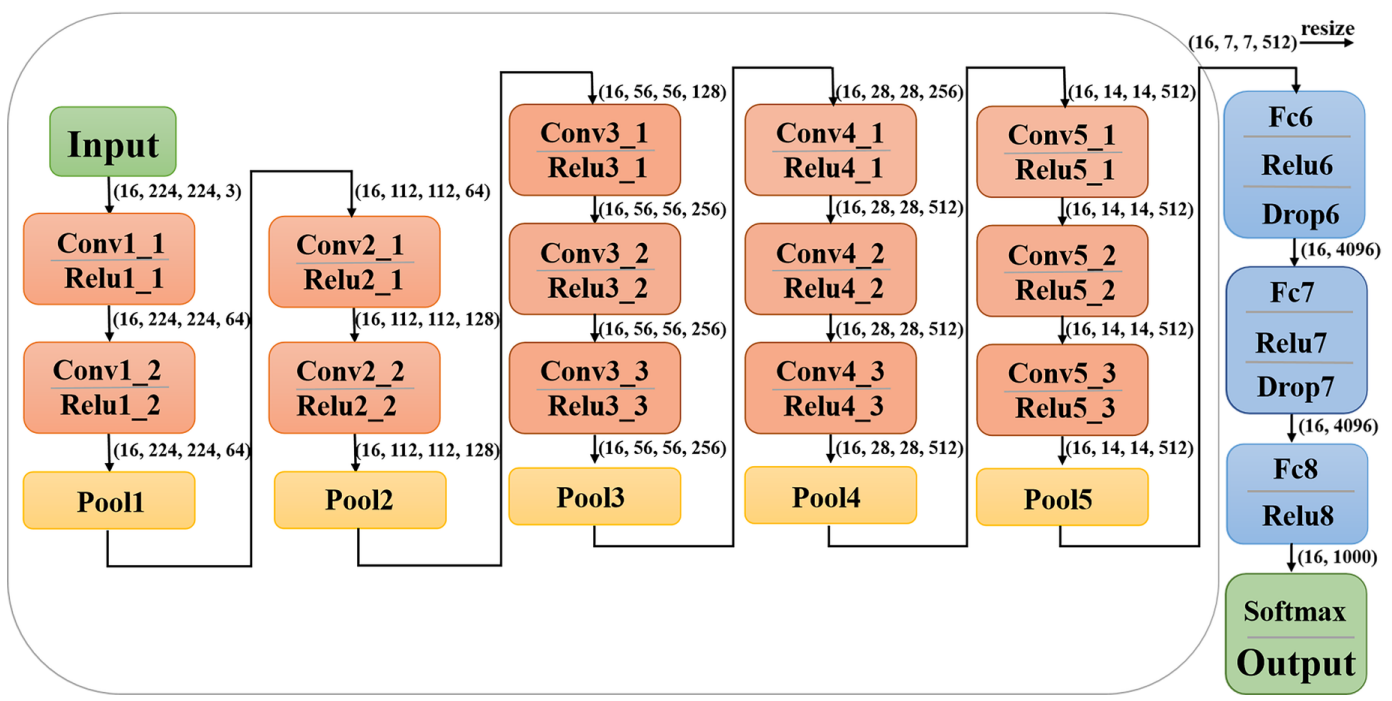




\subsubsection{GoogLeNet}

GoogLeNet (Szegedy et al., 2014), the winner of ILSVRC 2014, precisely aimed at how to effectively reduce the computational cost and complexity, which just possessed roughly 6.8 million parameters with nine inception modules, two convolutional layers, one convolutional layer for dimension reduction, two normalization layers, four max-pooling layers, one average pooling, one fully-connected layer, and a linear layer with softmax activation in the output. The whole architecture of GoogLeNet is stacked by inception modules that combine the multi-scale idea and dimension reduction layers based on the Hebbian principle and embedding learning. Each inception module inspired by the network in network uses a parallel combination of $1 \times 1,3 \times 3,5 \times 5$ convolutional layer along with $3 \times 3$ max pooling layer (see Figure 5), which contributes to increasing the width and depth of the network greatly, preventing over-fitting and more importantly improving accuracy. GoogLeNet also uses dropout regularization in the fully-connected layer and applies the ReLU activation function in all of the convolutional layers just like VGGNet. In our work, InceptionV3 is selected as a component of the MultiModel_VGR.

\subsubsection{ResNet}

ResNet (He et al., 2015) won the first place in ILSVRC 2015 and COCO 2015 classification challenge with error rate of $3.57 \%$, which introduced residual units for addressing the degradation problem in the case of deepening the network. ResNet is exactly stacked by many residual units, which can be expressed in formula as follows (He et al., 2016):

$$
\begin{aligned}
& y_{l}=h\left(x_{l}\right)+F\left(x_{l}, W_{l}\right) \\
& x_{l+1}=f\left(y_{l}\right)
\end{aligned}
$$

where $x_{l}$ and $x_{l+1}$ are input and output of the $l$ th unit, and $F$ is a residual function. In [13], $h\left(x_{l}\right)=x_{l}$ is an identity mapping and $f$ is a ReLU function. A building block in ResNet is designed to reduce the number of parameters (see Figure 6 ), where the first $1 \times 1$ convolution layer reduces the dimension from 256 to 64 , and the latter is responsible for recovery. For saving computing resources

Figure 5. A raw inception module of GoogLeNet

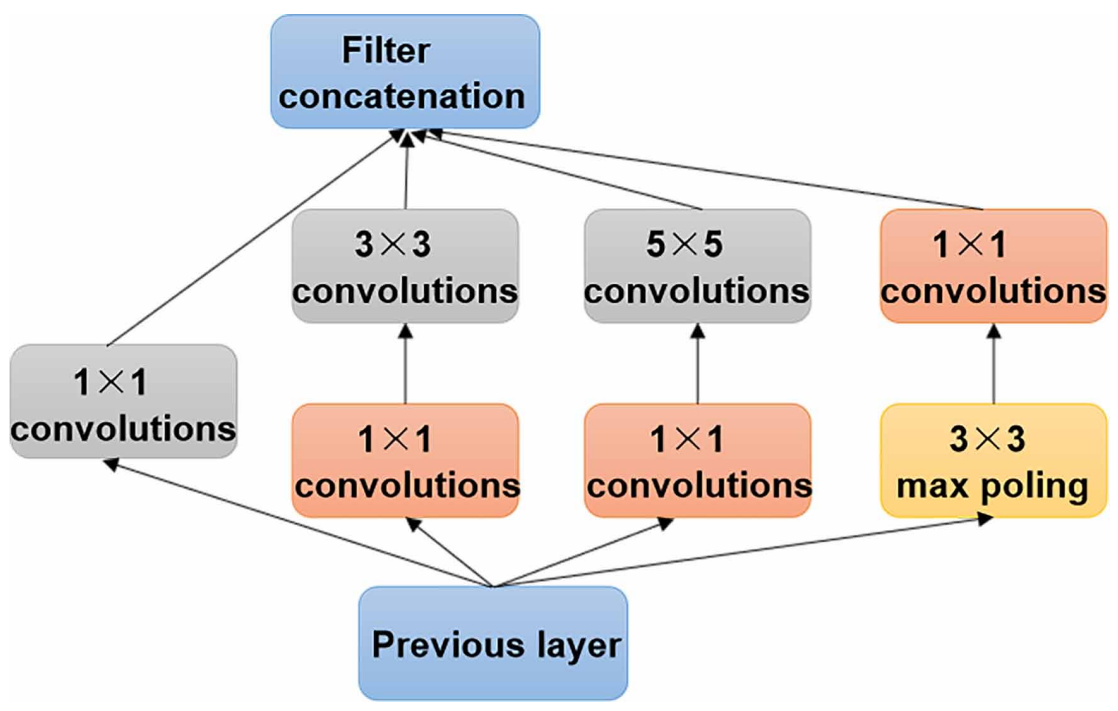


Figure 6 . A building block of ResNet

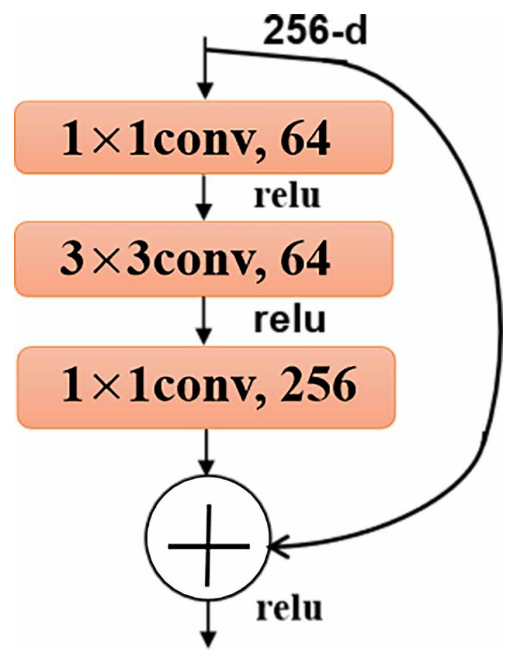

and training time, ResNet50 is chosen to be another basic network of the MultiModel_VGR, which also has high performance in various classification tasks.

\subsubsection{MultiModel_VGR}

Considering that the used dataset of pepper leaf disease infected by Xanthomonas campestris is relatively small and burden of parameters in CNNs is heavy, direct training from scratch is the last choice. Since large similarity between our dataset and ImageNet dataset, transfer learning rather than fine-tuning is adopted to boost classification performance and reduce training time, i.e. all the pretrained parameters on the ImageNet dataset in the convolution layers are frozen and not changed. As shown in Figure 7, the input data is resized to the expected input size of the respective CNNs and

Figure 7. The architecture of MultiModel_VGR

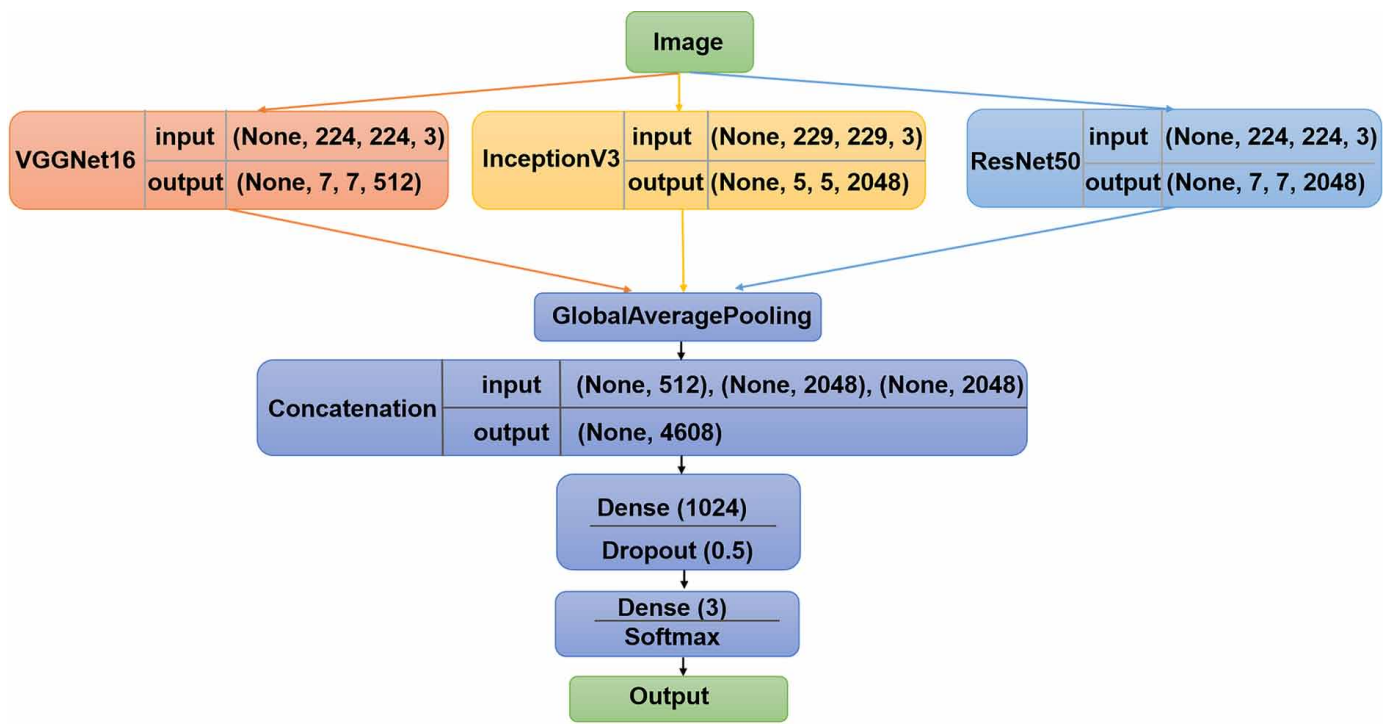


propagated forward to each basic single model, i.e. VGGNet16, InceptionV3 and ResNet50. The distinctive feature maps are extracted by multi-type convolution filters arranged in single CNNs. Through global average pooling (GAP), the feature maps extracted from convolution layers output in the form of two-dimensional matrix and then merged by the way of concatenation. Multiple CNNs generate discriminative feature maps and the united two-dimensional feature matrix combines, which improves the flexibility and characterization ability of the MultiModels. After features fusion, a fully connected layer, a dropout layer and a classification layer are added. Since the abundant training parameters and complex structures in the proposed MultiModels easily cause over-fitting, dropout layers are used and the rate is set to 0.5 . The softmax classifier is used as the final classification layer to compute the classification scores of the corresponding class.

\subsection{Experimental Setup}

The experimental setup is shown in Table 2, the experiments are performed on dual Graphics Processing Unit (GPU) mode to improve training speed.

The original architectures used for the automatic detection and severity assessment of VGGNet, GoogleNet and ResNet are trained as control experiments of the MultiModel_VGR to explore whether the integrated approach will improve the performance. In addition, the binary-integrated MultiModels, i.e. MultiModel_VG based on VGGNet16 and InceptionV3, MultiModel_VR based on VGGNet16 and ResNet50 and MultiModel_GR based on InceptionV3 and ResNet50 are also introduced to explore the influence of basic single models.

To enable a fair comparison between the results of all the experimental configurations, all the hyper-parameters are identical across all the experiments. The optimizer SGD is used with base learning rate 0.001 , weight decay $1 \mathrm{e}-6$ and momentum 0.9 to update parameters of the added lays, but all the parameters pre-trained on the ImageNet dataset in the convolution layers are frozen and not changed. While learning, the SGD algorithm works by randomly choosing a small number of training inputs referred to as batch size which is set to 16. Each of these experiments runs for a total of 100 epochs and the training process are recorded, where one epoch is defined as the number of training iterations in which the particular CNN has completed a full pass of the whole training dataset. Early-stopping mechanism is used to deal with the problem of over-fitting and speed up the training process. The training ends when validation loss does not display significant decline for a predefined number of epochs. In addition, the best model is saved and will be used for prediction and evaluation on test dataset.

\section{RESULTS AND DISCUSSION}

In order to explore the advantageous performance of the proposed integrated approach and to visualize and analysis the training process, validation process is conducted by setting the above hyper-parameters. The accuracy and loss against the number of epochs during training is illustrated in Figure 8. Since parameters of all the deep CNN models are pre-trained on ImageNet dataset, the loss curves go down from a very small initial value. The validation accuracy of all the experimental architectures increases steadily and the validation loss drops gradually with the growth of the epoch at the beginning. In early-stopping mode, VGGNet16, InceptionV3, ResNet50, MultiModel_VG,

Table 2. Experimental setup

\begin{tabular}{|l|l|}
\hline \multicolumn{1}{|c|}{ Hardware } & \multicolumn{1}{c|}{ Software } \\
\hline CPU: Intel Core i7-6700HQ & Windows10 \\
RAM: 8 GB DDR4 & CUDA9. 0 + CUDNN7. 0 \\
GPU: NVIDIA GEFORCE GTX 960M & Keras, Tensorflow \\
\hline
\end{tabular}


Figure 8. Validation accuracy and validation loss of different architectures are compared while the number of epoch is varied in the case of early-stopping mechanism. VGGNet, GoogleNet, ResNet, MultiModel_VG, MultiModel_VR, MultiModel_GR and MultiModel_VGR stop training with the epoch 23, 46, 39, 15, 25, 40, and 20, respectively.
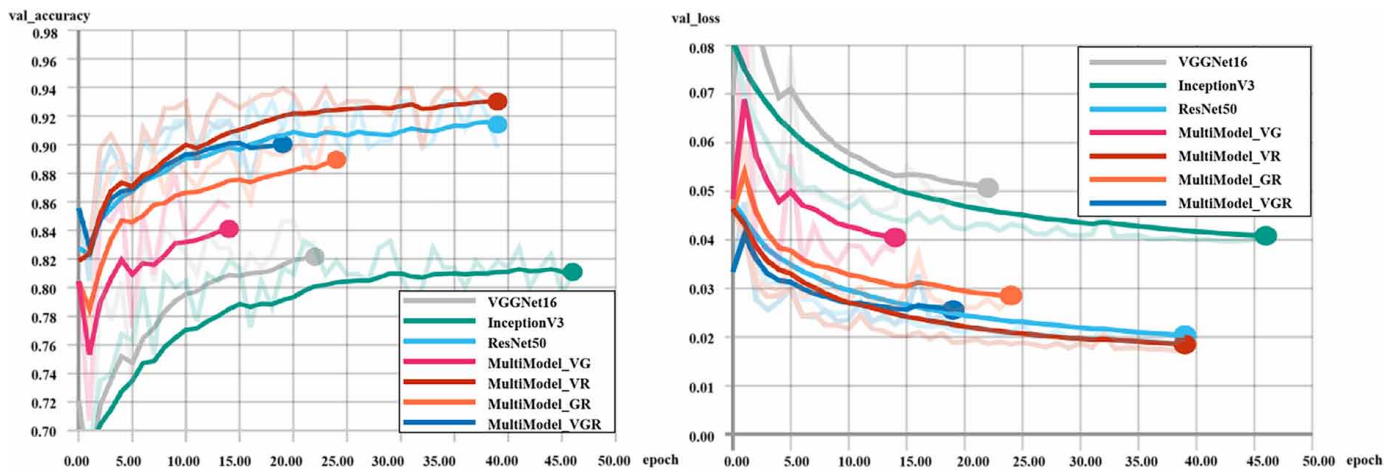

MultiModel_VR, MultiModel_GR and MultiModel_VGR stop training when the epoch equals to 23, 46, 39, 15, 25, 40 and 20 respectively. MultiModel_VGR converges quickly and shows no signs of performance deterioration compared with single CNN models and binary-integrated MultiModels.

Although ResNet50 and MultiModel_VR hold the lead in validation accuracy by a narrow margin at the expense of longer training epochs, MultiModel_VGR outperforms VGGNet16, InceptionV3, ResNet50, MultiModel_VG, MultiModel_VR, MultiModel_GR in test accuracy as shown in Figure 9. MultiModel_VGR achieves the highest test accuracy (95.34\%), which suggests the superiority of integrated CNNs.

Consistent with Figure 6, the average precision (95.49\%), recall (95.33\%) and F1-score $(95.37 \%)$ of MultiModel_VGR are the highest among all these models as shown in Table 3. And scores of InceptionV3 are the lowest with precision $(88.10 \%)$, recall $(87.33 \%)$ and F1-score $(87.37 \%)$. Noted that the integrated networks have different degrees of improvement compared with the single networks. But experimental results also show that this is not always the case and MultiModel_GR based on InceptionV3 and ResNet50 is a negative example. As with other integrated approaches, the choice of the basic single networks has no absolute rule and needs to be adapted to a specific dataset. In another thing, the integration is most effective when the basic models are significantly different, and their combination will enable the integrated network to capture more distinguishing feature information, thereby improving recognition accuracy.

For a more detailed comparison at different operating points, confusion matrices of different models on test samples are performed as shown in Figure 10. The threshold of probability is set

Figure 9. Accuracy of VGGNet16, InceptionV3, ResNet50, MultiModel_VG, MultiModel_GR, MultiModel_VG and MultiModel_VGR on test dataset

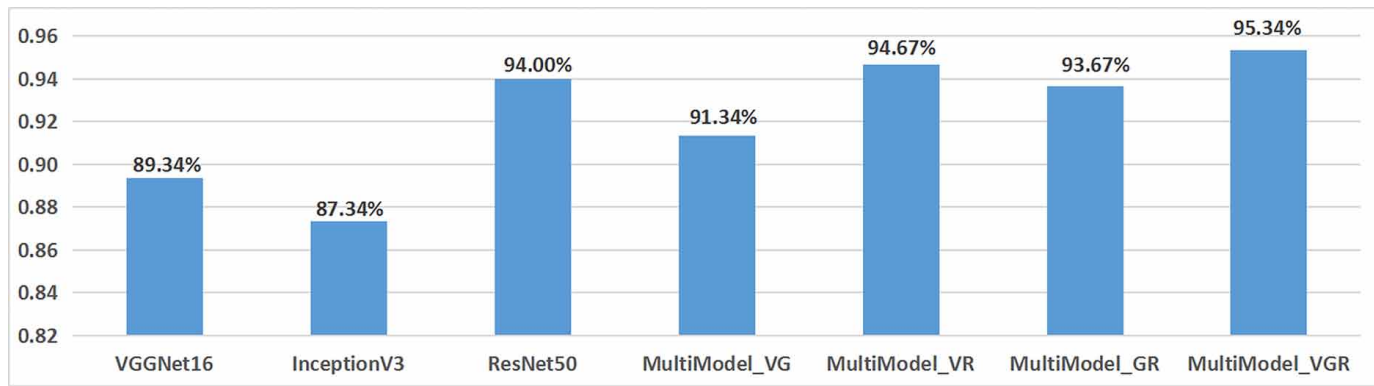


Table 3. Precision, recall and F1-score for the corresponding experimental configurations

\begin{tabular}{|c|c|c|c|c|c|c|}
\hline \multirow{2}{*}{ Architecture } & \multirow{2}{*}{ Criterion } & Label & Label1 & Label2 & Label3 & Total/Average \\
\hline & & Support & 100 & 100 & 100 & 300 \\
\hline \multirow{3}{*}{ VGGNet16 } & \multicolumn{2}{|l|}{ precision } & 0.8559 & 0.8542 & 0.9785 & 0.8962 \\
\hline & \multicolumn{2}{|l|}{ recall } & 0.9500 & 0.8200 & 0.9100 & 0.8933 \\
\hline & \multicolumn{2}{|l|}{ F1-score } & 0.9005 & 0.8367 & 0.9430 & 0.8934 \\
\hline \multirow{3}{*}{ InceptionV3 } & \multicolumn{2}{|l|}{ precision } & 0.8348 & 0.8200 & 0.9882 & 0.8810 \\
\hline & \multicolumn{2}{|l|}{ recall } & 0.9600 & 0.8200 & 0.8400 & 0.8733 \\
\hline & \multicolumn{2}{|l|}{ F1-score } & 0.8930 & 0.8200 & 0.9081 & 0.8737 \\
\hline \multirow{3}{*}{ ResNet50 } & \multicolumn{2}{|l|}{ precision } & 0.9515 & 0.9184 & 0.9495 & 0.9398 \\
\hline & \multicolumn{2}{|l|}{ recall } & 0.9800 & 0.9000 & 0.9400 & 0.9400 \\
\hline & \multicolumn{2}{|l|}{ F1-score } & 0.9655 & 0.9091 & 0.9447 & 0.9398 \\
\hline \multirow{3}{*}{ MultiModel_VG } & \multicolumn{2}{|l|}{ precision } & 0.9029 & 0.8700 & 0.9691 & 0.9140 \\
\hline & \multicolumn{2}{|l|}{ recall } & 0.9300 & 0.8700 & 0.9400 & 0.9133 \\
\hline & \multicolumn{2}{|l|}{ F1-score } & 0.9163 & 0.8700 & 0.9543 & 0.9135 \\
\hline \multirow{3}{*}{ MultiModel_VR } & \multicolumn{2}{|l|}{ precision } & 0.9500 & 0.9118 & 0.9796 & 0.9471 \\
\hline & \multicolumn{2}{|l|}{ recall } & 0.9500 & 0.9300 & 0.9600 & 0.9467 \\
\hline & \multicolumn{2}{|l|}{ F1-score } & 0.9500 & 0.9208 & 0.9697 & 0.9468 \\
\hline \multirow{3}{*}{ MultiModel_GR } & \multicolumn{2}{|l|}{ precision } & 0.9074 & 0.9263 & 0.9423 & 0.9377 \\
\hline & \multicolumn{2}{|l|}{ recall } & 0.9800 & 0.8800 & 0.9026 & 0.9367 \\
\hline & \multicolumn{2}{|l|}{ F1-score } & 0.9423 & 0.9500 & 0.9645 & 0.9364 \\
\hline \multirow{3}{*}{ MultiModel_VGR } & \multicolumn{2}{|l|}{ precision } & 0.9694 & 0.9057 & 0.9596 & 0.9549 \\
\hline & \multicolumn{2}{|l|}{ recall } & 0.9500 & 0.9600 & 0.9320 & 0.9533 \\
\hline & \multicolumn{2}{|l|}{ F1-score } & 0.9596 & 0.9500 & 0.9694 & 0.9537 \\
\hline
\end{tabular}

to 0.5 and the fraction of accurately predicted images for each class is displayed in detail. Note the color distribution of the confusion matrix and that the results of VGGNet16 and InceptionV3 are unsatisfactory. ResNet50 improves accuracy by a large margin compared with VGGNet16 and InceptionV3. For MultiModels, MultiModel_VGR obtains the accuracy rate of label1, label2, label3 with $95 \%, 96 \%$ and $95 \%$, respectively. General samples are prone to be misclassified. However, the misclassified samples are only confused with their adjacent labels. For example, the healthy samples are only confused with the general samples, and none of healthy samples are classified as serious samples. The large green area of healthy leaves and the brownish appearance of serious samples infected with Xanthomonas campestris make them easier to be distinguished. The confusion between adjacent labels is due to their similar pathological features and contributes a major share to the overall error. Compared with ResNet50, MultiModel_VGR improves the recognition accuracy of label3 but sacrifices the accuracy benefits in label1. For pepper leaves with no obvious pathological feature, i.e. general samples which are in early affected stage, ResNet50 tends to misclassify diseased samples as healthy, which is supposed to be avoided in actual agricultural production. MultiModel_VGR measures the extracted features in a more comprehensive point of view and makes a compromise. Different single algorithm models in MultiModels actually observe dataset from different data spatial and structural perspectives. On the basis of their own algorithmic principles, MultiModel_VGR extracts more discriminative leaf pathological features for pepper bacterial spot disease detection and 
Figure 10. Confusion matrices of VGGNet16, InceptionV3, ResNet50 and MultiModels on test dataset are illustrated with an accuracy associated for individual classes which are listed along the diagonal. The confusion matrices are given in terms of percentages, not absolute numbers and the color of the matrix block represents the number of corresponding test samples as shown in the color bar.

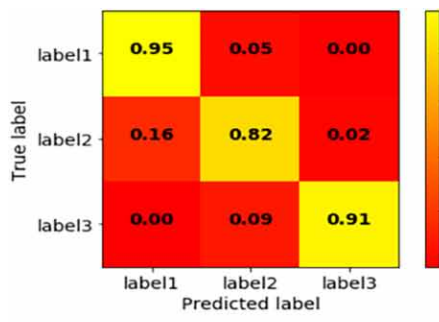

(a) VGGNet16

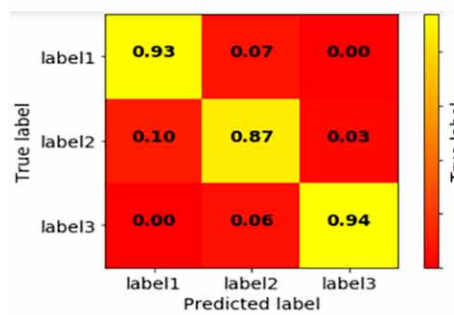

(d) MultiModel_vG

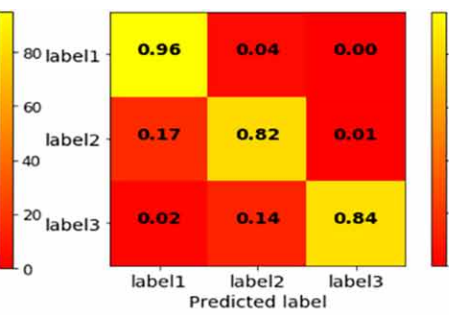

(b) Inception $\mathrm{V} 3$

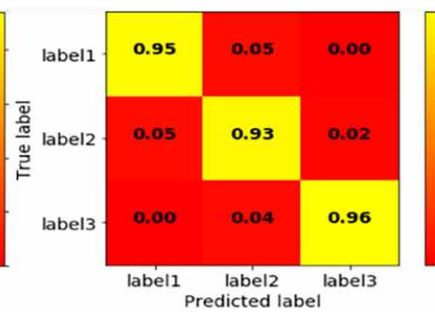

(e) MultiModel_VR

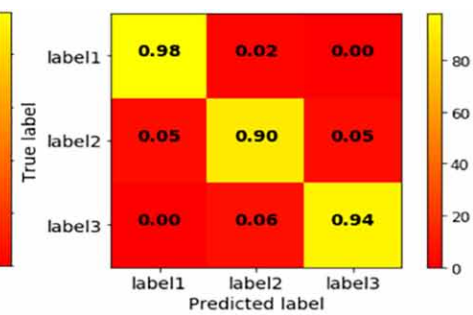

(c) ResNet50

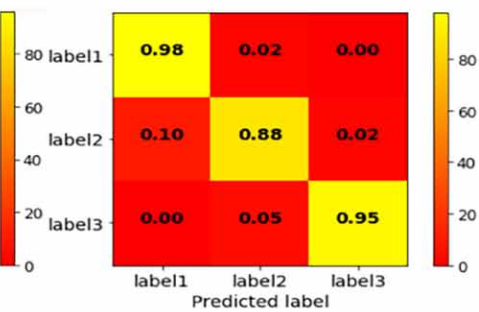

(f) MultiModel_GR

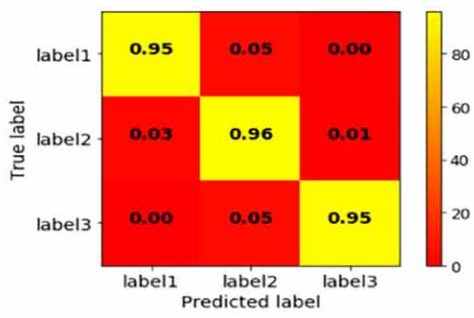

(g) MultiModel_vGR

severity assessment. It is this complementary principle that makes MultiModel_VGR obtain superior in performance and thus the representational ability is strengthened. In addition, the use of transfer learning makes this process more concise and efficient.

\section{CONCLUSION AND PERSPECTIVES}

This paper proposes the MultiModels to detect and assess severity of pepper leaf disease infected by Xanthomonas campestris through extracting more effective and discriminative features. MultiModel_ VGR fuses VGGNet16, InceptionV3 and ResNet50 and is designed to classify pepper leaf images into healthy samples (label1), general samples (label2) and serious samples (label3). The accuracy of each class and the overall accuracy on test dataset show that MultiModel_VGR outperforms the state-of-the-art single CNNs architectures including VGGNet16, InceptionV3 and ResNet50, which proves the effectiveness of integrated approach. The main advantage of MultiModels is complementary ability and enhanced representational ability. The experiments on binary-integrated MultiModels illustrate that the choice of the basic single networks has no absolute rule and needs to be adapted to a specific dataset. Since the collected dataset is small and our classification task involves finegrained analysis, transfer learning method, data argumentation techniques, train-validation-test scheme and early-stopping mechanism are applied to prevent over-fitting and enhance the robustness and generalization capabilities of the deep CNNs. 
Any scientific research is of value only when it is applied to practical purposes and serves human beings. Our results are the first step toward an automatic diagnosis system for pepper bacterial spot disease. Our future work will be focused on the choice of classifiers, basic single networks and more streamlined integrated approaches and other crop disease identification or similar visual recognition problems will be further explored.

\section{ACKNOWLEDGMENT}

This work was supported by the Public Welfare Industry (Agriculture) Research Projects Level-2 under Grant 201503116-04-06; Postdoctoral Foundation of Heilongjiang Province under Grant LBHZ15020; Harbin Applied Technology Research and Development Program under Grant 2017RAQXJ096 and National Key Application Research and Development Program in China under Grant 2018YFD0300105-2. 


\section{REFERENCES}

Cheng, X., Zhang, Y., Chen, Y., Wu, Y., \& Yue, Y. (2017). Pest identification via deep residual learning in complex background. Computers and Electronics in Agriculture, 141, 351-356. doi:10.1016/j.compag.2017.08.005

Geetharamani, G., \& Arun Pandian, J. (2019). Identification of plant leaf diseases using a nine-layer deep convolutional neural network. Computers \& Electrical Engineering, 76, 323-338. doi:10.1016/j. compeleceng.2019.04.011

Guan, W., Yu, S., \& Jianxin, W. (2017). Automatic image-based plant disease severity estimation using deep learning. Computational Intelligence and Neuroscience, 2017, 1-8. doi:10.1155/2017/2917536 PMID:28757863

Han, L., Haleem, M. S., \& Taylor, M. (2015). A novel computer vision-based approach to automatic detection and severity assessment of crop diseases. In Proceedings of the 2015 Science and Information Conference (SAI). IEEE. doi:10.1109/SAI.2015.7237209

He, K., Zhang, X., Ren, S., \& Sun, J. (2015). Deep residual learning for image recognition. In Proceedings of the IEEE Conference on computer vision and pattern recognition. IEEE Press. doi:10.1109/CVPR.2016.90

He, K., Zhang, X., Ren, S., \& Sun, J. (2016). Identity mappings in deep residual networks. In European conference on computer vision (pp. 630-645). Springer. doi:10.1007/978-3-319-46493-0_38

Hughes, D. P., \& Salathe, M. (2015). An open access repository of images on plant health to enable the development of mobile disease diagnostics.

Krizhevsky, A., Sutskever, I., \& Hinton, G. E. (2012). ImageNet classification with deep convolutional neural networks. In Proceedings of the International Conference on Neural Information Processing Systems. Academic Press. doi:10.1145/3065386

Kussul, N., Lavreniuk, M., Skakun, S., \& Shelestov, A. (2017). Deep learning classification of land cover and crop types using remote sensing data. IEEE Geoscience and Remote Sensing Letters, 14(5), 778-782. doi:10.1109/ LGRS.2017.2681128

Lecun, Y., Bottou, L., Bengio, Y., \& Haffner, P. (1998). Gradient-based learning applied to document recognition. Proceedings of the IEEE, 86(11), 2278-2324. doi:10.1109/5.726791

Li, G., Ma, Z., \& Wang, H. (2012). Image Recognition of Grape Downy Mildew and Grape Powdery Mildew Based on Support Vector Machine. In International Conference on Computer and Computing Technologies in Agriculture (pp. 151-162). Springer. doi:10.1007/978-3-642-27275-2_17

Liang, Q., Xiang, S., Hu, Y., Coppola, G., Zhang, D., \& Sun, W. (2019). Pd2se-net: Computer-assisted plant disease diagnosis and severity estimation network. Computers and Electronics in Agriculture, 157, 518-529. doi:10.1016/j.compag.2019.01.034

Lin, Z., Mu, S., Huang, F., Khattak, A. M., Wang, M., Gao, W., \& Jia, J. (2019). A united matrix-based convolutional neural network for fine-grained image classification of wheat leaf diseases. IEEE Access, 7 , 11570-11590. doi:10.1109/ACCESS.2019.2891739

Mohanty, S. P., Hughes, D. P., \& Salathé, M. (2016). Using deep learning for image-based plant disease detection. Frontiers in Plant Science, 7, 1419-1428. doi:10.3389/fpls.2016.01419 PMID:27713752

Philipp, L., Jens, B., Andres, M., \& Cyrill, S. (2018). Fully convolutional networks with sequential information for robust crop and weed detection in precision farming. IEEE Robotics and Automation Letters. doi:10.1109/ LRA.2018.2846289

Rahnemoonfar, M., \& Sheppard, C. (2017). Deep count: fruit counting based on deep simulated learning. Sensors, 17(4), 905. doi:10.3390/s17040905

Simonyan, K., \& Zisserman, A. (2014). Very deep convolutional networks for large-scale image recognition.

Sumbul, G., Cinbis, R. G., \& Aksoy, S. (2017). Fine-grained object recognition and zero-shot learning in remote sensing imagery. IEEE Transactions on Geoscience and Remote Sensing. doi:10.1109/TGRS.2017.2754648

Szegedy, C., Ioffe, S., Vanhoucke, V., \& Alemi, A. (2016). Inception-v4, inception-resnet and the impact of residual connections on learning. 
Szegedy, C., Liu, W., Jia, Y., Sermanet, P., Reed, S., Anguelov, D., \& Rabinovich, A. et al. (2015). Going deeper with convolutions. In Proceedings of the IEEE conference on computer vision and pattern recognition (pp. 1-9). IEEE Press. doi:10.1109/cvpr.2015.7298594

Wang, X., \& Cheng, C. (2016). Weed seeds classification based on PCANet deep learning baseline. In Proceedings of the 2015 Asia-Pacific Signal and Information Processing Association Annual Summit and Conference (APSIPA). Academic Press. doi:10.1109/APSIPA.2015.7415304

Xie, D., Lei, Z., \& Li, B. (2017). Deep learning in visual computing and signal processing. Applied Computational Intelligence and Soft Computing, 2017, 1-13. doi:10.1155/2017/1320780

Yu, Y., Jin, Q., \& Chen, C. W. (2018). FF-CMnet: A CNN-Based Model for Fine-Grained Classification of Car Models Based on Feature Fusion. In Proceedings of the 2018 IEEE International Conference on Multimedia and Expo (ICME). IEEE Computer Society. doi:10.1109/ICME.2018.8486443

Zhang, K., Xu, Z., Dong, S., Cen, C., \& Wu, Q. (2019b). Identification of peach leaf disease infected by Xanthomonas campestris with deep learning. In Engineering in Agriculture, Environment and Food. Academic Press. doi:10.1016/j.eaef.2019.05.001

Zhang, K., Zhang, L., \& Wu, Q. (2019a). Identification of cherry Leaf disease infected by Podosphaera Pannosa via convolutional neural network. International Journal of Agricultural and Environmental Information Systems, 10(2), 98-110. doi:10.4018/IJAEIS.2019040105

Zhang, L., Yang, F., Zhang, D., \& Zhu, Y. J. (2016). Road crack detection using deep convolutional neural network. In Proceedings of the IEEE International Conference on Image Processing (ICIP 2016) (pp. 3708-3712). IEEE Press. doi:10.1109/ICIP.2016.7533052

Zhou, Z., Shin, J., Zhang, L., Gurudu, S., \& Liang, J. (2017). Fine-Tuning Convolutional Neural Networks for Biomedical Image Analysis: Actively and Incrementally. In Proceedings of the IEEE Conference on Computer Vision and Pattern Recognition (CVPR). IEEE Press. doi:10.1109/CVPR.2017.506

Qiufeng Wu was born at Heilongjiang Province, China, in 1979. He received Ph.D in computer application technology from Harbin Institute of Technology, Harbin, China, in January 2014. He is working as associated professor in College of Arts and Sciences in Northeast Agricultural University. He is CCF member. His current research interests include machine learning, computer vision and smart agriculture.

Miaomiao Ji received her Bachelor's degree in Engineering Management from Xuzhou University of Technology, Xuzhou, China. She is currently a graduate student in Management Science and Engineering at Northeast Agricultural University, Harbin, China. Her current research interests focus on machine learning and intelligent agricultural.

Zhao Deng received a bachelor's degree in engineering cost from the Three Gorges University in 2013. Now studying for a master's degree in management science and engineering from Northeast Agricultural University. Her research interests include statistics, machine learning and intelligent transportation systems. 